\title{
Estimating the Potential for Reduction of Hospital Capacity at the Community Level
}

\author{
Ronald Lagoe*, Shelly Littau \\ Hospital Executive Council, Syracuse, NY, USA \\ Email: *Hospexcl@cnymail.com
}

Received 18 March 2016; accepted 26 April 2016; published 29 April 2016

Copyright (C) 2016 by authors and Scientific Research Publishing Inc.

This work is licensed under the Creative Commons Attribution International License (CC BY). http://creativecommons.org/licenses/by/4.0/

\section{(c) (i) Open Access}

\begin{abstract}
This study estimated the potential impact of the nationwide shift from inpatient to outpatient care in the hospitals of Syracuse, New York, a small metropolitan area with a relatively stable population. The study employed the $3 \mathrm{M}^{\mathrm{TM}}$ All Patients Refined Diagnosis Group Severity of Illness system to identify inpatients and related utilization with the greatest potential for movement from inpatient to outpatient settings. The study data suggested that the development of additional ambulatory care capacity in Syracuse could support the reduction of an average daily census of approximately 60 - 125 patients with low severity of illness, excluding readmissions. The study data also identified the potential for shifting an average daily census of approximately 9 - 19 patients who were readmitted to hospitals within 30 days of their initial admissions from inpatient to outpatient care. The study data also identified the potential for reduction of an average daily census of approximately 20 - 70 adult medicine and adult surgery patients through continued initiatives for inpatient length of stay reduction. The impact of initiatives in each of these areas could result in a reduction of the combined average daily adult medicine and adult surgery census of the Syracuse hospitals from approximately 90 to 215 patients. This would amount to between 8 and 20 percent of the current inpatient census for adult medicine and adult surgery. These data suggest that planning for initiatives such as ambulatory care development and reduction of readmissions should also include evaluation of their impact on inpatient acute care and related services.
\end{abstract}

\section{Keywords}

Hospital Utilization, Hospital Admissions, Hospital Readmissions, Hospital Lengths of Stay

\section{Introduction}

In the United States, increased attention is focusing on the improvement of the efficiency and outcomes of

${ }^{*}$ Corresponding author. 
health care. This attention has been supported by greater interest in improving care at the national, state, and community levels [1] [2].

Among hospitals in the United States and the communities that they serve, efforts to improve efficiency and outcomes will probably generate at least two major developments. One that is already in progress is a decline in hospital admissions. Another includes efforts to reduce lengths of stay and related resource consumption for remaining inpatients at high severity of illness [3] [4]. Both of these developments will reduce the need for inpatient hospital beds and related resources.

Efforts to eliminate the need for inpatient hospital beds are focusing on reducing admissions to acute hospitals. These initiatives include programs that shift low severity of illness patients from inpatient to outpatient settings and programs that reduce hospital readmissions. At the national level, initiatives such as Accountable Care Organizations, bundling of services, and Medicaid restructuring, are focusing on movement of hospital inpatients to ambulatory care [5] [6]. In recent years, the development of reimbursement penalties for excess readmissions has stimulated considerable interest in this subject. Many of these initiatives are being supported by health care payors because they save payments for inpatient hospitalization [5] [7]-[10].

Efforts to improve the efficiency and outcomes of care are also focusing on improving hospital utilization by reducing lengths of stay for hospital patients at high severity of illness. These patients are associated with extensive use of health care services and related expenses. They are sometimes known as superutilizers. As more patients at lower severity are shifted to other settings of care, these high severity patients will comprise the majority of hospital inpatient populations [11] [12].

All of these initiatives have the potential for reducing inpatient utilization in acute care hospitals. As a result, they will produce reduced need for hospital bed capacity of hospitals at the community level. They will also increase the severity of illness of remaining acute care populations.

\section{Population}

This study evaluated the potential impacts of hospital efforts that will reduce inpatient utilization in the metropolitan area of Syracuse, New York. This area includes three large acute care facilities, Crouse Hospital (19,776 discharges excluding well newborns, 2015), St. Joseph’s Hospital Health Center (28,237 discharges, 2015), and Upstate University Hospital (28,237 discharges, 2015). Within the immediate service area, the hospitals work with a combined medical staff of more than 1700 physicians and 12 nursing homes.

The immediate service area of the hospitals includes all of Onondaga County and portions of Cayuga, Cortland, Madison and Oswego Counties, with a population of approximately 600,000. The hospitals provide tertiary services to the eleven county Central New York Health Service Area with a population of approximately $1,400,000[13]$.

Since 1978, the Syracuse hospitals have worked cooperatively to improve the efficiency and outcomes of acute care and related services through the Hospital Executive Council. These efforts have included initiatives related to emergency medical services, inpatient acute care, hospital medical staffs, and long term care services [14] [15].

Historically, the service area of the Syracuse hospitals has maintained an inpatient admission rate that has been below national and state averages but slightly higher than those of New York State metropolitan areas such as Rochester and Albany. In 2014, the inpatient readmission rate for Syracuse was 4.0 percent higher than the rates for Rochester and Albany.

These initiatives have also stimulated efforts to improve the efficiency of care through length of stay reduction and outcomes management. These efforts have focused on reduction of inpatient lengths of stay for patients at major and extreme severity of illness through development of the potential to care for these patients in area nursing homes [15]. A number of these initiatives have developed through a partnership with $3 \mathrm{M}^{\mathrm{TM}}$ Health Information Systems involving Severity of Illness, Potentially Preventable Readmissions, and Potentially Preventable Complications data [10] [16] [17].

\section{Methods}

This study evaluated potential impacts of efforts to improve efficiency and outcomes on inpatient adult medicine and adult surgery utilization of the hospitals of Syracuse, New York. These services account for approximately 
70 percent of utilization in the Syracuse hospitals and a large percentage of hospital use in the United States. The study focused on expansion of ambulatory care, elimination of inpatient readmissions, and reduction on inpatient lengths of stay.

The first component of the study focused on the potential impact of efforts to reduce inpatient adult medicine and adult surgery admissions, excluding readmissions within 30 days of the initial admissions, in the Syracuse hospitals. The study data were used to identify numbers of inpatient discharges and patient days for adult medicine and adult surgery inpatients excluding readmissions within 30 days in the Syracuse hospitals in 2012, 2013, 2014, and 2015. Inpatient readmissions and related patient days were identified using the Potentially Preventable Readmissions software and subtracted from these data for subsequent analysis. The remaining inpatients and patient days were identified by All Patients Refined Severity of Illness so that patients with the lowest severity and the highest potential for care in ambulatory setting could be evaluated. These data were used to construct ranges based on the potential for moving 75 percent of remaining adult medicine and adult surgery inpatients at Minor severity of illness and 25 percent of remaining medical surgical inpatients at Moderate severity of illness to ambulatory care.

The second component of the study focused on the potential impact of efforts to reduce inpatient readmissions for adult medicine and adult surgery in the Syracuse hospitals. In Syracuse, approximately 90 percent of these rehospitalizations have involved adult medicine patients. The study data identified numbers of readmissions for adult medicine and adult surgery patients at Minor and Moderate severity of illness by year for 2013, 2014, and 2015 using the Potentially Preventable Readmissions System. Because of their lower severity of illness, these patients had the most potential for care outside inpatient hospitals.

The study data were used to construct ranges of inpatients for whom readmissions within 30 days of the initial admissions, could be avoided. The minimum levels of these ranges were based on eliminating 50 percent of readmissions and related patient days at minor and moderate severity of illness for 2014. The maximum levels of ranges were based on elimination of 100 percent of actual readmissions for these populations.

The third component of the study focused on the potential impact of reductions in inpatient lengths of stay for adult medicine and adult surgery in the Syracuse hospitals. During the past two decades, the Syracuse hospitals and the Hospital Executive Council have developed a number of initiatives to address this subject.

The study data were used to identify mean lengths of stay for adult medicine and adult surgery inpatients by severity of illness in the Syracuse hospitals for 2012, 2013, 2014, and 2015. The same data were also employed to identify differences in patient days for these services between mean lengths of stay in the Syracuse hospitals and the severity adjusted national averages. Based on this information and the experiences of the Syracuse hospitals, a range of patient days that could be eliminated through length of stay reduction was constructed.

\section{Results}

The first component of the study focused on the potential for movement of adult medicine and adult surgery inpatients, excluding inpatient readmissions, at low severity of illness to ambulatory care in the Syracuse hospitals. Relevant data are summarized in Table 1.

These data demonstrated that adult medicine and adult surgery discharges in the Syracuse hospitals excluding readmissions declined by 3.5 percent, from 51,931 to 50,125, between 2012 and 2015. During the same period, inpatient days associated with these discharges increased by 0.8 percent, from 280,948 to 281,162.

The data demonstrated that adult medicine and adult surgery patients at minor and moderate severity of illness in the Syracuse hospitals generated lower proportions of inpatient days, between 38.6 and 35.3 percent of the total, than patients at Major and Extreme severity between 2012 and 2015. These percentages declined slightly as the proportion related to higher severity of illness patients increased. The changes reflected the impact of limited efforts to shift low severity of illness inpatients to ambulatory care settings during the recent years.

Projection of the potential impact of increased use of ambulatory care on inpatient adult medicine and adult surgery utilization in the Syracuse hospitals was based on the implementation of additional outpatient programs by the area's largest acute care providers, Upstate University Hospital and St. Joseph's Hospital Health Center as well as the development of an Accountable Care Organization, Medicare bundling initiatives, and other population based efforts.

Based on this information, as well as the differences in discharges per 1000 population among New York State metropolitan areas identified previously, it was estimated that most adult medicine discharges and adult surgery discharges at minor severity of illness and some discharges at moderate severity of illness for these services 
Table 1. Inpatient adult medical/surgical discharges, patient days, and readmissions, by severity of illness, Syracuse Hospitals, 2012-2015.

\begin{tabular}{|c|c|c|c|c|c|c|c|c|}
\hline & \multicolumn{4}{|c|}{ Discharges } & \multicolumn{4}{|c|}{ Patient Days } \\
\hline & 2012 & 2013 & 2014 & 2015 & 2012 & 2013 & 2014 & 2015 \\
\hline \multicolumn{9}{|c|}{ Severity of Illness } \\
\hline Extreme & 5243 & 5663 & 5599 & 6107 & 77,336 & 79,150 & 81,980 & 90,923 \\
\hline Major & 16,788 & 17,066 & 16,843 & 16,931 & 111,890 & 111,745 & 113,485 & 110,027 \\
\hline Moderate & 21,249 & 20,414 & 20,527 & 20,428 & 82,313 & 78,227 & 80,403 & 77,775 \\
\hline Minor & 12,428 & 11,376 & 11,012 & 10,741 & 33,097 & 29,776 & 30,436 & 28,446 \\
\hline \multirow[t]{3}{*}{ Total } & 55,708 & 54,519 & 53,981 & 54,207 & 304,636 & 298,898 & 306,304 & 307,171 \\
\hline & \multicolumn{4}{|c|}{ Discharges Excluding Readmissions } & \multicolumn{4}{|c|}{ Patient Days Excluding Readmission Days } \\
\hline & 2012 & 2013 & 2014 & 2015 & 2012 & 2013 & 2014 & 2015 \\
\hline \multicolumn{9}{|c|}{ Severity of Illness } \\
\hline Extreme & 4733 & 5021 & 4968 & 5441 & 70,941 & 72,350 & 74,694 & 83,606 \\
\hline Major & 15,183 & 15,195 & 15,163 & 15,088 & 101,537 & 99,950 & 102,792 & 98,327 \\
\hline Moderate & 20,014 & 19,154 & 19,338 & 19,193 & 76,826 & 72,757 & 74,954 & 71,970 \\
\hline Minor & 12,001 & 10,968 & 10,594 & 10,403 & 31,644 & 28,297 & 28,904 & 27,259 \\
\hline Total & 51,931 & 50,338 & 50,063 & 50,125 & 280,948 & 273,354 & 281,344 & 281,162 \\
\hline
\end{tabular}

Data exclude diagnosis related groups concerning obstetrics, pediatrics, psychiatry, alcohol/substance abuse treatment, rehabilitation, and all patients aged 0 - 17 years. Source: Hospital Executive Council.

excluding readmissions in the Syracuse hospitals could be eliminated through treatment in ambulatory care settings. The low end of this range was based on elimination of 75 percent of adult medicine and adult surgery discharges at minor severity of illness. The high end was based on elimination of 25 percent of discharges at minor severity and 40 percent of discharges at moderate severity. Using the data for 2012 and 2015, this range would include elimination of an average daily census of 56.0 - 64.9 patients at the minimum and 124.0 - 138.5 at the maximum.

The second component of the study focused on the potential impact of avoiding adult medicine and adult surgery readmissions within 30 days of the initial admissions in the Syracuse hospitals. Relevant data are summarized in Table 2.

These data demonstrated that adult medicine and adult surgery readmissions within 30 days in the Syracuse hospitals increased by 8.1 percent, from 3777 to 4082, between 2012 and 2015. During the same period, inpatient days associated with these readmissions increased by 9.8 percent, from 23,688, to 26,009, accounting for an average daily census of 64.7 to 71.3 inpatients.

The data related to readmission rates indicated that adult medicine and adult surgery readmission rates within 30 days in the Syracuse hospitals were considerably higher for patients at major and extreme severity (12 - 18 percent), than those for patients at minor and moderate severity (3 - 7 percent). This information suggested that lower severity readmissions had greater potential for being addressed without inpatient care.

The study data also demonstrated that adult medicine and adult surgery patients at Minor and Moderate severity of illness in the Syracuse hospitals generated lower proportions of inpatient days, 29.2 - 26.9 percent, between 2012 and 2015 than patients at major and extreme severity. Based on this information, the number of inpatient days generated by inpatient readmissions for adult medicine and adult surgery patients at Minor and moderate severity of illness combined in the Syracuse hospitals ranged from 6940 in 2012 to 6992 in 2015. This amounted to an average daily census of 19.0 to 19.2 patients. Elimination of readmissions for 50 percent of these patients would have reduced the average daily census by 9.5 to 9.6 patients, while elimination of all of these readmissions would have reduced the average daily census by 19.0 - 19.2 patients. 
Table 2. Inpatient adult medical-surgical readmissions, by severity of illness, Syracuse hospitals, 2012-2015.

\begin{tabular}{|c|c|c|c|c|}
\hline & \multicolumn{4}{|c|}{ Number of Readmissions } \\
\hline & 2012 & 2013 & 2014 & 2015 \\
\hline \multicolumn{5}{|l|}{ Severity of Illness } \\
\hline Extreme & 510 & 642 & 631 & 666 \\
\hline Major & 1605 & 1871 & 1680 & 1843 \\
\hline Moderate & 1235 & 1260 & 1189 & 1235 \\
\hline Minor & 427 & 408 & 418 & 338 \\
\hline Total & 3777 & 4181 & 3918 & 4082 \\
\hline Severity of Illness & \multicolumn{4}{|c|}{ Readmission Patient Days } \\
\hline Extreme & 6395 & 6800 & 7286 & 7317 \\
\hline Major & 10,353 & 11,795 & 10,693 & 11,700 \\
\hline Moderate & 5487 & 5470 & 5449 & 5805 \\
\hline Minor & 1453 & 1479 & 1532 & 1187 \\
\hline Total & 23,688 & 25,544 & 24,960 & 26,009 \\
\hline Severity of Illness & \multicolumn{4}{|c|}{ Readmission Rates } \\
\hline Extreme & 16.09 & 16.18 & 18.60 & 18.18 \\
\hline Major & 12.17 & 12.30 & 12.73 & 13.66 \\
\hline Moderate & 7.23 & 6.84 & 7.00 & 6.74 \\
\hline Minor & 3.98 & 3.84 & 4.43 & 3.35 \\
\hline Total & 8.55 & 8.67 & 9.11 & 8.96 \\
\hline
\end{tabular}

Source: Hospital Executive Council.

The third component of the analysis focused on the impact of reductions in inpatient length of stay reduction on inpatient utilization in the Syracuse hospitals. Relevant data are summarized in Table 3.

These data demonstrated that the longest stays for adult medicine and adult surgery patients in the Syracuse hospitals involved patients at major and extreme severity of illness. Between 2012 and 2015, mean stays for patients at Extreme severity of illness were more than twice the stays for all patients for these services, while mean stays for patients at Major severity of illness were approximately 20 percent longer than those of the total population.

The study data also demonstrated that, on average, all of the excess patient days for adult medicine and adult surgery patients in the Syracuse hospitals during the four year period were associated with patients at major and extreme severity of illness. For patients at extreme severity of illness, these excess days ranged from 468.51 to 7969.6 or an average daily census of 1.3 to 21.8 . For patients at major and extreme severity of illness combined, the number of excess patient days ranged from 2473.11 to $12,523.66$, or an average daily census of 6.8 to 34.2 .

It should be noted that these data reflect the impact of length of stay reduction programs implemented by the Syracuse hospitals and the Hospital Executive Council between 2012 and 2015. Most of this impact involved the Complex Care programs with area nursing homes that were implemented in 2015. It should also be noted that mean stays for adult medicine and adult surgery patients in the hospitals remained above severity adjusted national averages throughout the period.

In order to evaluate the potential impact of subsequent length of stay reduction initiatives on inpatient utilization for adult medicine and adult surgery in the Syracuse hospitals, a range was constructed based on excess patient days for major and extreme severity of illness. The minimum patient days of this range was based on the remaining days and average daily census for Major and Extreme severity of illness patients for 2012-2015. The 
Table 3. Inpatient adult medical-surgical discharges and patient days, by Severity of illness, Syracuse hospitals, 2012-2015.

\begin{tabular}{cccccccccc}
\hline & \multicolumn{3}{c}{ Mean Lengths of Stay } & \multicolumn{5}{c}{ Excess Patient Days } \\
\cline { 2 - 9 } & 2012 & 2013 & 2014 & 2015 & 2012 & 2013 & 2014 & 2015 \\
\hline Severity of Illness & & & & & & & & & \\
Extreme & 14.75 & 13.98 & 14.64 & 14.89 & 7969.60 & 3328.56 & 8088.95 & 468.51 \\
Major & 6.66 & 6.55 & 6.74 & 6.50 & 4554.06 & 2212.12 & 4243.35 & 2004.60 \\
Moderate & 3.87 & 3.83 & 3.92 & 3.81 & -366.60 & -1521.92 & -1076.92 & -427.35 \\
Minor & 2.66 & 2.62 & 2.76 & 2.65 & -1461.04 & -1918.53 & -1108.76 & -468.15 \\
Total & 5.47 & 5.48 & 5.67 & 5.67 & $10,863.62$ & 2180.01 & $10,205.65$ & 1455.39 \\
\hline
\end{tabular}

Data exclude diagnosis related groups concerning obstetrics, pediatrics, psychiatry, alcohol/substance abuse treatment, rehabilitation, and all patients aged 0 - 17 years. Source: Hospital Executive Council.

maximum of this range was based on elimination of the minimum level of inpatient days, plus additional utilization equal to the number of excess days for major and extreme severity of illness patients in 2012 and 2014. This level would bring mean lengths of stay for major and extreme severity of illness patients below the severity adjusted national average. Using these calculations, subsequent length of stay reduction programs were estimated to result in the elimination of an adult medicine and adult surgery average daily census between 7 - 34 and 77.5 patients.

\section{Discussion}

Current experience in the health care system of the United States includes extensive efforts to improve efficiency and outcomes. Many of these efforts concern reduction of hospital inpatient care. Some of them involve partnerships between providers and payers such as Accountable Care Organizations and bundling of services. Their impact is already resulting in the shift of health care capacity from inpatient to ambulatory care services.

These characteristics make this health system an excellent environment for predicting the impact of these developments.

The study employed the $3 \mathrm{M}^{\mathrm{TM}}$ All Patients Refined Diagnosis Related Group Severity of Illness system to identify hospital inpatients and related utilization with the greatest potential for movement from inpatient to outpatient settings. This system is based on a nationally validated algorithm that includes extensive clinical and demographic information.

The development of additional ambulatory care capacity in Syracuse could support the reduction of an average daily census of approximately 60 - 125 patients with low severity of illness, excluding readmissions. It is being stimulated, in part, by the development of Accountable Care Organizations, a major state effort to restructure care for Medicaid patients and bundling of services. Additional ambulatory care capacity is being developed in inner city areas with the highest hospital admission rates. Some of these areas have inpatient admission rates 50 - 80 percent higher than the area average.

The study data also identified the potential for shifting an average daily census of approximately 9 - 19 patients who were readmitted to hospitals within 30 days of their initial admissions from inpatient to outpatient care. This utilization would involve diverting readmissions with low severity of illness to outpatient care.

The study data also identified the potential for reduction of an average daily census of approximately 20 - 70 adult medicine and adult surgery patients through continued initiatives for inpatient length of stay reduction. The hospitals have achieved notable progress in this area during recent years; however, additional opportunities remain through cooperative programs with local long term care facilities.

From a planning standpoint, the impact of initiatives in each of these areas could result in a reduction of the combined average daily adult medicine and adult surgery census of the Syracuse hospitals from approximately 90 to 215 patients. This would amount to between 8 and 20 percent of the current inpatient census for adult medicine and adult surgery. It would depend on the impact of a number of initiatives related to utilization and outcomes.

It should also be noted that patients who received medical observation without inpatient admission were 
omitted from these estimates. If this population, which comprised an average daily census of about 65 patients, were included, the estimated reduction would still amount to an average daily census of 8 - 18 percent.

\section{Conclusions}

These data suggest that planning for initiatives such as ambulatory care development and reduction of readmissions should also include evaluation of their impact on inpatient acute care and related services. In the Syracuse metropolitan area, these data would not be offset by population immigration from other areas.

As the health care system of the United States continues to change, the movement of patients from inpatient to outpatient services will probably receive increased attention. Additional research can help further define important issues related to this subject such as the effects of specific programs, the characteristics of patients involved, and the impact of these developments on health care costs.

\section{References}

[1] Dentzler, S. (2011) Urgent Measures for an Old Problem. Health Affairs, 30, 1626. http://dx.doi.org/10.1377/hlthaff.2011.0961

[2] Kellermann, A.D.L. (2011) A Decade of Health Care Cost Growth Has Wiped out Real Income Gains for an Average U.S. Family. Health Affairs, 30, 1630-1636. http://dx.doi.org/10.1377/hlthaff.2011.0585

[3] Weil, A.R. (2015) Hospital Cost and Quality. Health Affairs, 34, 1263. http://dx.doi.org/10.1377/hlthaff.2015.0786

[4] Weil, A.R. (2016) High Cost Populations, the ACA, and More. Health Affairs, 35, 7. http://dx.doi.org/10.1377/hlthaff.2015.1562

[5] Rose, S., Zaslavsky, A.M. and McWilliams, J.M. (2016) Variation in Accountable Care Organization Spending and Sensitivity to Risk Adjustment: Implications for Benchmarking. Health Affairs, 35, 440-448. http://dx.doi.org/10.1377/hlthaff.2015.1026

[6] Colla, C.H., Lewis, V.A. and Tierney, E. (2016) Hospitals Participating in ACOs Tend to Be Large and Urban. Allowing Access to Capital and Data, 35, 431-439.

[7] Goozner, M. (2016) Searching for Green Shoots at HIMMS Conference. Modern Healthcare, 46, 24.

[8] Pasely, B.H., Lagoe, R.J. and Marshall, N.O. (1995) Excess Acute Care Bed Capacity and Its Causes: The Experience of New York State. HSR: Health Services Research, 30, 115-131.

[9] Lagoe, R.J. (1998) Benchmarking Admissions Identifies Areas for Improvement. Healthcare Benchmarks, 5, 89-91.

[10] Goldfield, N.J., McCullough, E.C., Hughes, J.S., Tang, A.M., Eastman, B., Rawlins, L.K. and Averill, R.F. (2008) Identifying Potentially Preventable Readmissions. Health Care Financing Review, 30, 75-92.

[11] Gawande, A.A. (2011) The Hot Spotters: Can We Lower Costs by Getting the Neediest Patients Better Care? New Yorker, 40-51.

[12] Hong, C.S., Siegel, A.L. and Ferris, T.G. (2014) Caring for High Need, High Cost Patients: What Makes a Successful Care Management Program? Commonwealth Fund, New York.

[13] Lagoe, R.J., Pasinski, T., Kronenberg, P., Quinn, T. and Schaengold, P. (2006) Linking Health Services at the Community Level. Canada Healthcare Quarterly, 9, 60-65. http://dx.doi.org/10.12927/hcq..18229

[14] Lagoe, R.J. and Westert, G.P. (2010) Evaluation of Hospital Inpatient Complications: A Planning Approach. BMC Health Services Research, 10, 200. http://dx.doi.org/10.1186/1472-6963-10-200

[15] Lagoe, R., Pernisi, L., Haag, D. and Drapola, B. (2015) Evolution of Programs to Improve Transfers between Hospitals and Nursing Homes at the Community Level. Open Journal of Nursing, 5, 587-595. http://dx.doi.org/10.4236/ojn.2015.57062

[16] $3 \mathrm{M}^{\mathrm{TM}}$ Health Information Systems (1998) All Patients Refined Diagnosis Related Groups (APR DRGs). M Health Information Systems 3, Wallingford, Conn.

[17] Hughes, J.S., Verill, R.F. and Goldfield, N.J. (2006) Identifying Potentially Preventable Complications Using a Present on Admissions Indicator. Health Care Financing Review, 27, 63-82. 